\title{
Enhanced prenatal case management for low income smokers
}

\author{
Patricia Dolan-Mullen, Carlo C DiClemente, Mary M Velasquez, Sandra C Timpson, \\ Janet Y Groff, Joseph P Carbonari, Laurel Nicol
}

Smoking during pregnancy is highly prevalent in populations of white, low socioeconomic status women who are more addicted to nicotine than most other groups within their social class. Qualitative and quantitative assessments conducted by this research team suggested that effective pregnancy smoking interventions should be offered in the context of high risk counselling that also addressed sources of stress, depression, negative family support, and the various problems associated with poverty. ${ }^{1-3}$ Because many of these women are in early stages of change for smoking cessation, they need an approach focused on motivation and decision making rather than advice or quitting guides. Motivational interviewing is an approach that addresses the motivational enhancement needs of these women, meets them at their stage of change, and therefore seemed particularly appropriate for these women. ${ }^{4}$

This project proposed using the existing prenatal high risk case management delivery system for motivational enhancement based smoking cessation counselling. The case manager's role is to give support and marshal resources to address emotional and environmental stressors for high risk pregnancies, a role supported by Medicaid in most states. In addition, we believed that the interaction style and counselling skills of case managers in the cooperating clinic network were compatible with the demands of an individualised, comprehensive, motivational approach to pregnancy smoking cessation.

\section{Methods}

The effectiveness of enhanced case management was to be tested using a single blind, historical comparison cohort (comparison group) and an intervention cohort (intervention group) recruited from the same 10 clinics in East Texas. The comparison group was recruited first and received the existing case management care that mentioned but did not focus on smoking cessation. After a brief washout and training period, the intervention group was recruited and scheduled to receive our enhanced case management protocol that was initiated in the clinics as a new standard care protocol delivered by the same case managers. Both groups were measured at the first prenatal visit and in late pregnancy. Inclusion criteria included $>15$ years of age, gestational age $<27$ weeks, English speaker, receiving case management services, and smoked $<8$ days ago. Smoking levels were determined by ques- tionnaire and urine cotinine samples at baseline and late pregnancy.

Enhanced case management was planned to integrate a unique combination of clinic based intervention strategies aimed at individual and family levels of change: (1) individually tailored feedback delivered by the case manager at the second prenatal care visit, using brief transtheoretical model measures developed in another Smoke Free Families (SSF) grant (JP Carbonari, et al, personal communication) and collected at the first prenatal visit; (2) motivational interviewing counselling at the feedback session and at 2-4 follow up visits to promote smoking cessation during pregnancy; and (3) reinforcing take home materials, including materials to promote family change.

Case managers were nurses and social workers in 10 urban and rural perinatal care clinics throughout East Texas. Our protocol required them to understand the process of change and the measures that were used to assess and provide feedback for their intervention group clients. A two day training program with continuing education credit was organised to teach the protocol, motivational interviewing strategies for engaging the client, how to deliver the feedback and how to keep smoking cessation as a topic in future visits.

Both groups were recruited to the "Pregnancy, stress, and smoking study," for measurement only. Standard and enhanced case management represented "usual care" during the time periods their respective groups received prenatal care. Thus, we decided, and the respective institutional review boards concurred, that the trial did not need to be described as an intervention study.

\section{Results}

The intervention program was fully developed, and all 20 clinic case managers were trained. Both groups were recruited, although not at the projected levels. In all, 154 women were recruited to the comparison group and 142 to the intervention group- $72 \%$ and $85 \%$ consent rates, respectively. The historical cohort design was successful in that the two groups did not differ significantly on the baseline measures (table 1). The average score on the mental health measure (MHI-5) ${ }^{5}$ was 53 $(\mathrm{SD}=22)$, almost exactly equal to the score of a national sample of women aged 25-34 years seeing mental health providers, and below the average score of women aged 25-34 years in a general US sample $(\mathrm{m}=72, \mathrm{SD}=19) .^{6}$ 
Table 1 Baseline variables by study group

\begin{tabular}{|c|c|c|c|c|}
\hline & \multicolumn{2}{|c|}{ Comparison } & \multicolumn{2}{|c|}{ Intervention } \\
\hline & $\%$ & $m(S D)$ & $\%$ & $m(S D)$ \\
\hline \multicolumn{5}{|l|}{ Maternal age (years) } \\
\hline$<21$ & 50.6 & & 42.3 & \\
\hline $22-30$ & 35.7 & & 40.8 & \\
\hline$>30$ & 35.0 & & 16.9 & \\
\hline \multicolumn{5}{|l|}{ Race/ethnicity } \\
\hline African American & 5.8 & & 9.2 & \\
\hline Hispanic & 1.3 & & 2.8 & \\
\hline White & 92.9 & & 90.1 & \\
\hline Other & 1.3 & & 0.7 & \\
\hline Married/living with partner & 60.4 & & 53.5 & \\
\hline Private Health insurance & 4.6 & & 2.8 & \\
\hline Gestational age (weeks) & & $11.6(5.8)$ & & $11.7(5.9)$ \\
\hline Primiparous & 46.4 & & 37.3 & \\
\hline Pregnancy unwanted & 22.1 & & 28.2 & \\
\hline Mental Health Index- 5 & & $54.1(21.5)$ & & $51.5(23.5)$ \\
\hline \multicolumn{5}{|l|}{ Stage for pregnancy cessation } \\
\hline Precontemplation & 22.4 & & 30.3 & \\
\hline Contemplation & 46.7 & & 41.5 & \\
\hline Preparation & 30.9 & & 28.2 & \\
\hline Cigarettes per day & & $12.2(9.2)$ & & $12.9(8.3)$ \\
\hline$<31$ minutes to first cigarette & 46.1 & & 62.0 & \\
\hline Urine cotinine value (median) & $(n=131)$ & 3680 & $(n=123)$ & 2510 \\
\hline
\end{tabular}

The proportions lost to follow up before the end of pregnancy were similar- $42 \%$ of the comparison group and $37 \%$ of the intervention group (after reducing the denominators for one maternal and three fetal deaths in the comparison group and three fetal deaths in the intervention group). The main reasons for these rates differed: two thirds of those lost to follow up in the comparison group left the clinic (versus $85 \%$ in the intervention group) and almost one third were missed by the clinic for their eighth month measure (versus $10 \%$ in the intervention group). Women lost to follow up did not differ from those with eighth month measures, except that completers were more likely to be precontemplators ( $31 \%$ v 20\%) and less likely to be in preparation $(26 \% v$ $35 \%)$ for pregnancy cessation $(p=0.05)$.

Exposure to the intervention was limited. Based on clinic visits with and without case management, of those with an eighth month measure, no more than $38 \%$ could have received the criterion $>2$ motivational interviewing sessions; 44\%, 1 session; and $17 \%$ no session. Further, observations of the case managers indicated that performance often did not meet our criteria for the motivational interviewing style. ${ }^{7}$ Thus, no betweengroup differences were expected at the eighth month, and none were found. Comparing the 55 women in the comparison group and 36 in the intervention group who had both urine samples and questionnaires at the eighth month, and including the 35 in the comparison group and 54 in the intervention group who had not left the clinic but who did not have a urine sample (classified as smokers, regardless of self report), the two groups had the same percentage of cessation- $2.2 \%$ (both $2 / 90)$. The criterion cotinine value was $<80 \mathrm{ng} / \mathrm{ml}$.

Discussion

Development of the intervention components and training was our most significant accomplishment. Systemic barriers and chang- ing health care policies made delivery of the intervention problematic, however. With 25\% of the recruited population in the precontemplation stage and in view of the low level of cessation, we believe that the intensive enhanced case management intervention developed for this trial is appropriate and should be tested in an efficacy study where intervention delivery is assured.

In our enthusiasm to contribute to the SFF initiative and confidence in the perinatal outreach clinic system, we did not foresee how turbulent the transition to managed care for Medicaid beneficiaries would become during the pilot test. We proceeded based on a best case scenario, with insufficient project time for developmental testing of the delivery system and insufficient financial resources to maintain control over implementation when the clinics were forced to reduce case management activities in favour of medical services. On the day the grant was awarded, the medical school based clinic network was given many small rural clinics previously under the direction of the state health department. These additional clinics had to be incorporated without additional funding or personnel, creating a serious burden for clinic staff and budgets. This proved to be a difficult and chaotic period, resulting in high staff turnover.

Another event in the external environment also disrupted clinic operations. Start-up of a Medicaid managed care pilot study in the area gave many clinic patients unprecedented access to private care. In some clinics half of those who initiated prenatal care transferred to a private physician. The clinics responded by reducing the maximum time spent waiting for or receiving services. This made the intervention time unwelcome. Case managers, most of whom are nurses, were reassigned to medical services. In short, the clinics were beset with changes and problems that had a major impact on our ability to recontact and follow recruited women and importantly, on intervention delivery.

Provision of small personal monetary incentives to case managers to recruit study participants and provide the intervention was not effective in the face of major system changes. This intervention should be tested in an efficacy study, with intervention delivery assured by using case managers who are selected, supervised, and paid by the project.

Lastly, from qualitative data we learned that saliva rather than urine is a better choice for a biologic measure to keep the samples from being thrown out in the clinic laboratory and to avoid the suspicion by study participants that urine might be tested for illegal drugs.

This grant was funded under the Robert Wood Johnson Foundation Smoke Free Families Program. We gratefully acknowledge the contributions of Gaye Cummins, MA (project director), Karen Cheng, MPH and Donna White, MPH (research assistants) of the University of Texas-Houston Health Science Center School of Public Health; Katherine Golden Beck, RN, MS (director, case management), Carolyn Nelson-Becker, MSN, CNM (director), and the clinic direlyn and case managers of the University of Texas Medical Branch at and case managers of the University of Texas
Galveston Regional Perinatal Care Program. 
1 Ershoff DH, Mullen PD, Quinn VP. A randomized trial of a serialized self-help smoking cessation program for pregnant women in an HMO. Am $\mathcal{F}$ Public Health 1989;

2 Hartmann KE, Thorp JM, Pahel-Short L, Koch MA. A randomized controlled trial of smoking cessation intervention in pregnancy in an academic clinic. Obstet Gynecol 1996;87:621-6.

3 Mullen PD, Pollak KI, Groff JY, DiClemente CC. Relations among psychosocial variables, addiction, and self-efficacy in lower and higher income pregnant smokers. Presented at the Society for Research on Nicotine and Tobacco, March 1999, San Diego.
4 Miller WR, Rollnick S. Motivational interviewing: preparing people to change addictive behavior. New York: Guilford people to chang

5 Berwick DM, Murphy JM, Goldman PA, Ware JE, Barsky AJ, Weinstein MC. Performance of a five-item mental health screening test. Med Care 1991;29.169-76.

6 Ware JE, Snow, KK, Kosinski M, et al. SF-36 health survey manual and interpretation guide. Boston: New England Medical Center, 1993.

7 Velasquez MM, Hecht J, Quinn V, Emmons K, DiClemente CC, Dolan-Mullen P. Application of motivational interviewing to prenatal smoking cessation: training and implementation issues. Tobacco Control 2000;9(suppl III):iii36-40.

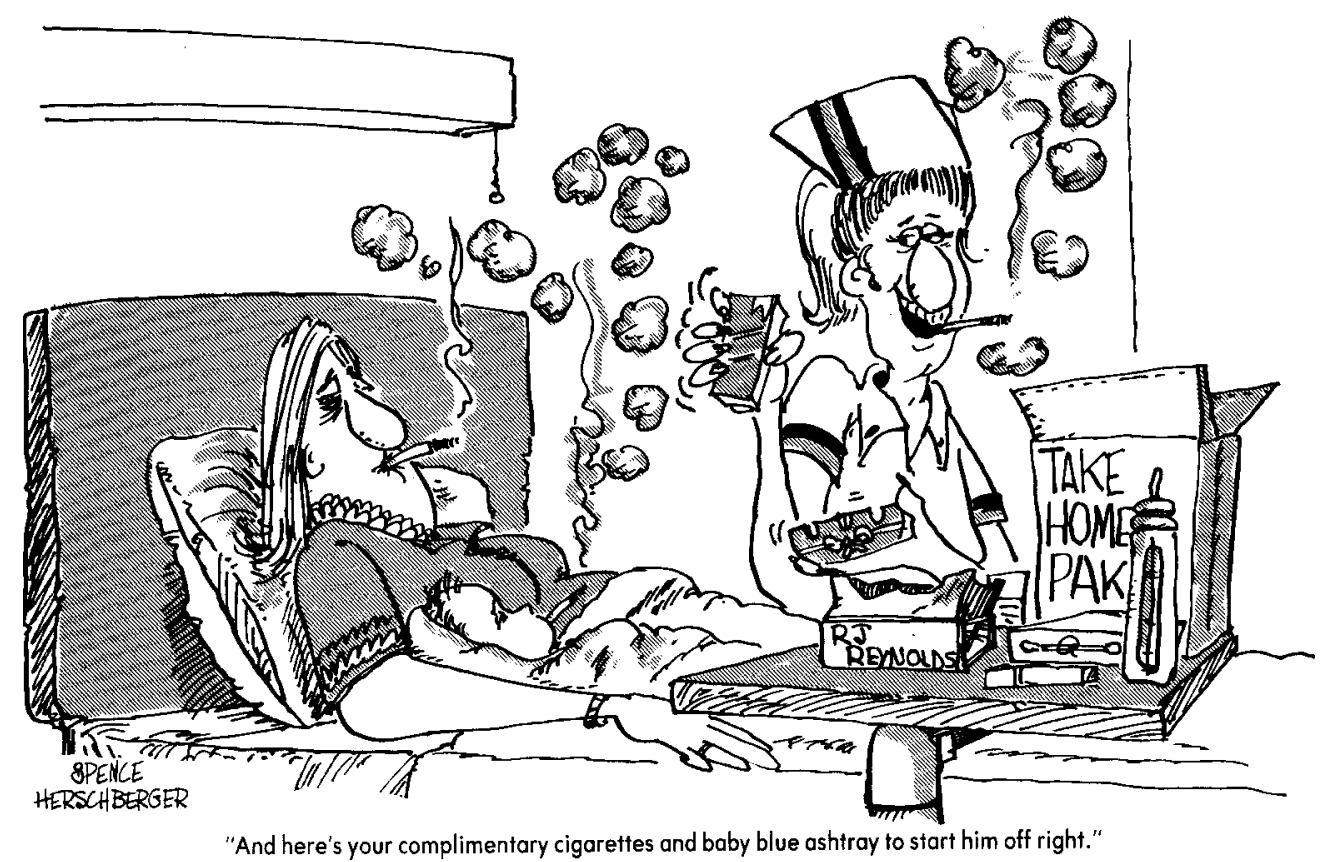

By WR Spence and V Herschberger, reprinted with permission of HEALTH EDCO, INC, Waco, Texas, USA. 